\title{
A GENERATOR FOR A SEMIGROUP OF NONLINEAR TRANSFORMATIONS
}

\author{
DOROTHY RUTLEDGE ${ }^{1}$
}

Let $S$ be a finite-dimensional Hilbert space and $T$ a function from $[0, \infty)$ to the set of continuous transformations from $S$ to $S$ satisfying

Condition (1) $T(0)=I$, and $T(x) T(y)=T(x+y)$ if $x, y \geqq 0$,

Condition (2) if $p$ is in $S$ and $g_{p}(x)=T(x) p$ for all $x \geqq 0$, then $g_{p}$ is continuous,

Condition (3) for each $x \geqq 0, T(x)$ is nonexpansive $(\|T(x) p-T(x) q\|$ $\leqq\|p-q\|$ for all $p$ and $q$ in $S$ ),

Condition (4) $S$ contains a rest point $r$ (i.e., $T(x) r=r$ for all $x \geqq 0$ ).

For each $\delta>0$, let $A_{\delta}=(1 / \delta)[T(\delta)-I]$. For each $p$ in $S$ for which $\lim _{\delta \rightarrow 0} A_{\delta} p$ exists, let $A p=\lim _{\delta \rightarrow 0} A_{\delta} p$.

It is well known that even for $S$ infinite-dimensional, if the semigroup $\{T(x) \mid x \geqq 0\}$ is linear (i.e., each $T(x)$ is linear), then the function $A$, called the infinitesimal generator of the semigroup, is defined on a dense subset of $S$, and for each $p$ in $S$, and each $x \geqq 0$, $\lim _{n \rightarrow \infty}[I-(x / n) A]^{-n} p=T(x) p$. (See, for example, [1].)

In [2] and [3], J. W. Neuberger considered semigroups similar to the ones considered in this paper, with the following condition assumed:

Condition (5) there is a dense subset $D$ of $S$ such that if $p$ is in $D$, then the derivative $g_{p}^{\prime}$ is continuous with domain $[0, \infty)$. In [3] he gave the following result, which will be used in a proof in this paper.

Theorem 1. Suppose $S$ is a normed linear complete space and Conditions (1), (2), (3), and (5) are satisfied. If $p$ is in $S$ and $x>0$ and $\epsilon>0$, there is a positive number $\delta$ such that if $0<y \leqq x$ and $0=t_{0}<t_{1}<\ldots$ $<t_{n+1}=y$ and $\left|t_{i+1}-t_{i}\right|<\delta$ for $i=0,1,2, \cdots, n$, then

$$
\lim _{\delta_{0}, \delta_{1}, \cdots, \delta_{n} \rightarrow 0}\left\|\prod_{i=0}^{n}\left[I-\left(t_{i+1}-t_{i}\right) A_{\delta_{i}}\right]^{-1} p-T(y) p\right\|<\epsilon .
$$

The purpose of this paper is to define a set $\left\{I_{x} \mid x \geqq 0\right\}$ of functions in terms of the functions $A_{\delta}$ in such a way that the functions $I_{x}$ generate the semigroup. The main results follow.

Presented to the Society, January 24, 1967; received by the editors November 20, 1967.

1 This paper is part of the author's Doctoral dissertation at Emory University, prepared under the supervision of J. W. Neuberger. 
THEOREM 2. If $\left\{\delta_{i}\right\}_{i=1}^{\infty}$ is a sequence of positive numbers converging to 0 , then there is a subsequence $\left\{\epsilon_{i}\right\}_{i=1}^{\infty}$ of $\left\{\delta_{i}\right\}_{i=1}^{\infty}$ such that if $x \geqq 0$ and $p$ is in $S$, then $\left\{\left(I-x A_{\epsilon_{i}}\right)^{-1} p\right\}_{i=1}^{\infty}$ converges to a point in $S$. For such a sequence $\left\{\epsilon_{i}\right\}_{i=1}^{\infty}$, if $x \geqq 0$, let $I_{x}$ be the function from $S$ into $S$ defined by

$$
I_{x p}=\lim _{n \rightarrow \infty}\left(I-x A_{\epsilon_{n}}\right)^{-1} p
$$

for each $p$ in $S$. Then each of the following is true.

(i) $\left\|I_{x} p-I_{x} q\right\| \leqq\|p-q\|$ for all $x \geqq 0$ and all $p$ and $q$ in $S$.

(ii) If $x>0$, then $\lim _{y \rightarrow x} I_{u} p=I_{x} p$.

(iii) If Condition (5) is satisfied, then $\lim _{n \rightarrow \infty}\left(I_{y / n}\right)^{n} p=T(y) p$ for all $y>0$ and all $p$ in $S$.

This theorem may be compared to a result found recently by Shinnosuke Ôharu [4], who considered $S$ to be a Banach space and assumed conditions which implied Conditions (1), (2), (3), and (5). For a nonlinear semigroup with these properties he found the following.

THEOREM 3. Let $\{T(x) \mid x \geqq 0\}$ be a semigroup as described above, and let $A$ be the infinitesimal generator such that for some $x_{0}>0$, the range of $I-x_{0} A$ is dense in $S$. Then for every $x>0$, there exist the function $(I-x A)^{-1}$ and its unique extension $L(x)$ onto $S$, which is nonexpansive, and $T(x)$ is represented by

$$
\lim _{n \rightarrow \infty} L(x / n)^{n} p=T(x) p
$$

for each $x \geqq 0$ and each $p$ in $S$.

If a semigroup satisfies the assumptions for both Theorem 3 and (iii) of Theorem 2, then $I_{x}=L(x)$ for $x \geqq 0$. However, Theorem 2 does not assume that for some $x>0$, the range of $I-x A$ is dense in $S$, and also Theorem 2 does not state that the collection $\left\{I_{x} \mid x \geqq 0\right\}$ is unique.

The proof of Theorem 2 will be developed by a sequence of lemmas, for which $S$ is finite-dimensional and Conditions (1)-(4) are assumed.

Lemma 1. If $p$ is in $S$ and $x>0$ and $\left\{\delta_{i}\right\}_{i=1}^{\infty}$ is a sequence of positive numbers, then there is a subsequence $\left\{\epsilon_{i}\right\}_{i=1}^{\infty}$ of $\{\delta\}_{i=1}^{\infty}$ such that $\left\{\left(I-x A_{\epsilon_{i}}\right)^{-1} p\right\}_{i=1}^{\infty}$ converges to a point in $S$.

Proof. In [2], Neuberger has a short proof that for $x>0$ and $\delta>0$, $\left(I-x A_{\delta}\right)^{-1}$ exists, has domain $S$, and is nonexpansive.

Now there is a rest point $r$ in $S$, and thus for $\delta>0,\left(I-x A_{\delta}\right)^{-1} r=r$. Thus 


$$
\left\|r-\left(I-x A_{\delta}\right)^{-1} p\right\|=\left\|\left(I-x A_{\delta}\right)^{-1} r-\left(I-x A_{\delta}\right)^{-1} p\right\| \leqq\|r-p\| .
$$

Then the set $\left\{\left(I-x A_{\delta_{i}}\right)^{-1} p \mid i=1,2, \cdots\right\}$ is bounded, and since $S$ is finite-dimensional, the lemma follows.

LEMMA 2. If $x>0$ and $\left\{\delta_{i}\right\}_{i=1}^{\infty}$ is a sequence of positive numbers converging to 0 , then there is a subsequence $\left\{\epsilon_{i}\right\}_{i=1}^{\infty}$ of $\left\{\delta_{i}\right\}_{i=1}^{\infty}$ such that for all $p$ in $S,\left\{\left(I-x A_{e_{i}}\right)^{-1} p\right\}_{i=1}^{\infty}$ converges.

Proof. Let $K=\left\{p_{1}, p_{2}, \cdots\right\}$ be a dense subset of $S$. By Lemma 1 , there is a subsequence $\left\{\delta_{1 i}\right\}_{i=1}^{\infty}$ of $\left\{\delta_{i}\right\}_{i=1}^{\infty}$ such that $\left\{\left(I-x A_{\delta_{1 i}}\right)^{-1} p_{1}\right\}_{i=1}^{\infty}$ converges. Continuing, for each $n>1$, a subsequence $\left\{\delta_{n i}\right\}_{i=1}^{\infty}$ of $\left\{\delta_{(n-1) i}\right\}_{i=1}^{\infty}$ can be obtained such that $\left\{\left(I-x A_{\delta_{n i}}\right)^{-1} p_{n}\right\}_{i=1}^{\infty}$ converges. Consider the subsequence $\left\{\delta_{i i}\right\}_{i=1}^{\infty}$ of $\left\{\delta_{i}\right\}_{i=1}^{\infty}$. It is easily seen that for each $p_{n}$ in $K,\left\{\left(I-x A_{\delta_{i i}}\right)^{-1} p_{n}\right\}_{i=1}^{\infty}$ converges to the sequential limit of $\left\{\left(I-x A_{\delta_{n i}}\right)^{-1} p_{n}\right\}_{i=1}^{\infty}$.

For each positive integer $i$, let $\epsilon_{i}=\delta_{i i}$. Then for all $q$ in $K$, $\left\{\left(I-x A_{\epsilon_{i}}\right)^{-1} q\right\}_{i=1}^{\infty}$ converges. Suppose $p$ is in $S$. If $\epsilon>0$, there is some $q$ in $K$ such that $\|p-q\|<\epsilon / 3$. There is some positive integer $N$ such that if $n>N$ and $m>N$, then $\left\|\left(I-x A_{\epsilon_{n}}\right)^{-1} q-\left(I-x A_{\epsilon_{m}}\right)^{-1} q\right\|<\epsilon / 3$. Since $\|p-q\|<\epsilon / 3$, it follows that $\left\|\left(I-x A_{\epsilon_{n}}\right)^{-1} p-\left(I-x A_{\epsilon_{n}}\right)^{-1} q\right\|$ $<\epsilon / 3$ and $\left\|\left(I-x A_{\epsilon_{m}}\right)^{-1} p-\left(I-x A_{\epsilon_{m}}\right)^{-1} q\right\|<\epsilon / 3$. It follows that $\left\|\left(I-x A_{\epsilon_{n}}\right)^{-1} p-\left(I-x A_{\epsilon_{m}}\right)^{-1} p\right\|<\epsilon$, and thus $\left\{\left(I-x A_{\epsilon_{i}}\right)^{-1} p\right\}_{i=1}^{\infty}$ is a Cauchy sequence and hence converges to a point in $S$. The lemma is proved.

Lemma 3. Suppose $Q$ is a countable subset of $(0, \infty)$ and $\left\{\delta_{i}\right\}_{i=1}^{\infty}$ is a sequence of positive numbers converging to 0 . Then there is a subsequence $\left\{\epsilon_{i}\right\}_{i=1}^{\infty}$ of $\left\{\delta_{i}\right\}_{i=1}^{\infty}$ such that for each $x$ in $Q$ and each $p$ in $S$, $\left\{\left(I-x A_{\epsilon_{i}}\right)^{-1} p\right\}_{i=1}^{\infty}$ converges to a point in $S$.

Proof. Let $Q=\left\{x_{1}, x_{2}, \cdots\right\}$ be a subset of $(0, \infty)$. By Lemma 2, there is a subsequence $\left\{\delta_{1 i}\right\}_{i=1}^{\infty}$ of $\left\{\delta_{i}\right\}_{i=1}^{\infty}$ such that $\left\{\left(I-x_{1} A_{\delta_{1 i}}\right)^{-1} p\right\}_{i=1}^{\infty}$ converges for all $p$ in $S$. Then there is a subsequence $\left\{\delta_{2 i}\right\}_{i=1}^{\infty}$ of $\left\{\delta_{1 i}\right\}_{i=1}^{\infty}$ such that $\left\{\left(I-x_{2} A_{\delta_{2 i}}\right)^{-1} p\right\}_{i=1}^{\infty}$ converges for all $p$ in $S$. By continuing this process, which is similar to that used in the proof of Lemma 2, one can show that the subsequence $\left\{\delta_{i i}\right\}_{i=1}^{\infty}$ of $\left\{\delta_{i}\right\}_{i=1}^{\infty}$ has the property that for every $x$ in $Q$ and every $p$ in $S,\left\{\left(I-x A_{\delta_{i i}}\right)^{-1} p\right\}_{i=1}^{\infty}$ converges.

Lemma 4. Suppose $p$ is in $S$. If $\delta>0$, let $F_{\delta}$ be the function from $[0, \infty)$ into $S$ defined by $F_{\delta}(x)=\left(I-x A_{\delta}\right)^{-1} p$. Then $F_{\delta}$ is continuous.

Proof. Let $x \geqq 0$. Let $M$ be a positive number greater than $\left\|A_{\delta}\left(I-x A_{\delta}\right)^{-1} p\right\|$. Let $\epsilon>0$. Let $y \geqq 0$ such that $|y-x|<\epsilon / M$. Then 


$$
\begin{aligned}
\left\|F_{\delta}(y)-F_{\delta}(x)\right\| & =\left\|\left(I-y A_{\delta}\right)^{-1} p-\left(I-x A_{\delta}\right)^{-1} p\right\| \\
& =\left\|\left(I-y A_{\delta}\right)^{-1} p-\left(I-y A_{\delta}\right)^{-1}\left(I-y A_{\delta}\right)\left(I-x A_{\delta}\right)^{-1} p\right\| \\
& \leqq\left\|p-\left(I-y A_{\delta}\right)\left(I-x A_{\delta}\right)^{-1} p\right\| \\
& =\left\|\left(I-x A_{\delta}\right)\left(I-x A_{\delta}\right)^{-1} p-\left(I-y A_{\delta}\right)\left(I-x A_{\delta}\right)^{-1} p\right\| \\
& =|y-x|\left\|A_{\delta}\left(I-x A_{\delta}\right)^{-1} p\right\|<\epsilon .
\end{aligned}
$$

Lemma 5. If $p$ is in $S$ and $c>0$, then the set

$$
\left\{\left\|A_{\delta}\left(I-x A_{\delta}\right)^{-1} p\right\| \mid x \geqq c, \delta>0\right\}
$$

is bounded above.

Proof. From the proof of Lemma 1, it is easily seen that the set $\left\{\left\|\left(I-x A_{\delta}\right)^{-1} p\right\| \mid x \geqq 0, \delta>0\right\}$ is bounded above. Let $M>0$ be an upper bound to this set. If $x \geqq c$ and $\delta>0$, then

$$
\begin{aligned}
\| A_{\delta}(I-x & \left.A_{\delta}\right)^{-1} p \| \\
& =(1 / x)\left\|-x A_{\delta}\left(I-x A_{\delta}\right)^{-1} p\right\| \\
& =(1 / x)\left\|\left(I-x A_{\delta}\right)\left(I-x A_{\delta}\right)^{-1} p-\left(I-x A_{\delta}\right)^{-1} p\right\| \\
& =(1 / x)\left\|p-\left(I-x A_{\delta}\right)^{-1} p\right\| \leqq(1 / c)\left[\|p\|+\left\|\left(I-x A_{\delta}\right)^{-1} p\right\|\right] \\
& \leqq(1 / c)[\|p\|+M] .
\end{aligned}
$$

LemMA 6. Suppose $p$ is in $S$ and for each $x \geqq 0, F_{\delta}(x)=\left(I-x A_{\delta}\right)^{-1} p$. Then the set $\left\{F_{\delta} \mid \delta>0\right\}$ is equicontinuous on $(0, \infty)$.

Proof. Let $x>0$. By Lemma 5 , there is an upper bound $M>0$ to the set $\left\{\left\|A_{\delta}\left(I-x A_{\delta}\right)^{-1} p\right\| \mid \delta>0\right\}$. Let $\epsilon>0$. If $y$ is a positive number such that $|x-y|<\epsilon / M$, then by the same argument as that used in the proof of Lemma $4,\left\|F_{\delta}(x)-F_{\delta}(y)\right\|<\epsilon$ for all $\delta>0$.

LEMMA 7. If $\left\{\delta_{i}\right\}_{i=1}^{\infty}$ is a sequence of positive numbers converging to 0 , there is a subsequence $\left\{\epsilon_{i}\right\}_{i=1}^{\infty}$ of $\left\{\delta_{i}\right\}_{i=1}^{\infty}$ such that for each $x \geqq 0$ and each $p$ in $S,\left\{\left(I-x A_{\epsilon_{i}}\right)^{-1} p\right\}_{i=1}^{\infty}$ converges to a point in $S$.

Proof. Let $Q$ be a countable dense subset of $(0, \infty)$. By Lemma 3 , there is a subsequence $\left\{\epsilon_{i}\right\}_{i=1}^{\infty}$ of $\left\{\delta_{i}\right\}_{i=1}^{\infty}$ such that for all $x$ in $Q$ and all $p$ in $S,\left\{\left(I-x A_{\epsilon_{i}}\right)^{-1} p\right\}_{i=1}^{\infty}$ converges. It will be shown that for all $x \geqq 0$ and all $p$ in $S$, this sequence converges.

For each $q$ in $S$ and each positive integer $i$, let

$$
F_{q, i}(x)=\left(I-x A_{\epsilon_{i}}\right)^{-1} q
$$

for all $x \geqq 0$. Let $p$ be in $S$. From Lemma 6 , the set $\left\{F_{p, i} \mid i=1,2, \cdots\right\}$ is equicontinuous on $(0, \infty)$. Also, for each $z$ in the dense subset $Q$ 
of $(0, \infty)$, the sequence $\left\{F_{p, i}(z)\right\}_{i=1}^{\infty}$ converges. Thus it follows that for every $x$ in $(0, \infty)$, the sequence $\left\{F_{p, i}(x)\right\}_{i=1}^{\infty}$ is a Cauchy sequence and hence converges to some point in $S$. That is, for every $x>0$, $\left\{\left(I-x A_{\epsilon_{i}}\right)^{-1} p\right\}_{i=1}^{\infty}$ converges. Since for $x=0$ the sequence clearly converges, the lemma is true, and the first statement in Theorem 2 is proved.

Consider the sequence $\left\{\epsilon_{i}\right\}_{i=1}^{\infty}$ given in the proof of Lemma 7 and consider the functions $F_{q, i}$ defined in the proof. For each $x \geqq 0$ and each $p$ in $S$, let $I_{x} p$ be the sequential limit of $\left\{\left(I-x A_{\epsilon_{i}}\right)^{-1} p\right\}_{i=1}^{\infty}$. For each $p$ in $S$, let $G_{p}$ be the function from $[0, \infty)$ into $S$ defined by $G_{p}(x)=I_{x} p$, so that for each $x \geqq 0, G_{p}(x)$ is the sequential limit of $\left\{F_{p, i}(x)\right\}_{i=1}^{\infty}$. These functions will be used in Lemmas 8 and 9 below.

LemMA 8. For each $x \geqq 0, I_{x}$ is nonexpansive.

Proof. Let $x \geqq 0$, and let each of $p$ and $q$ be in $S$. If $\epsilon>0$ there is a positive integer $n$ such that $\left\|\left(I-x A_{\epsilon_{n}}\right)^{-1} p-I_{x} p\right\|<\epsilon / 2$ and $\left\|\left(I-x A_{\epsilon_{n}}\right)^{-1} q-I_{x} q\right\|<\epsilon / 2$. From this it follows that

$$
\left\|I_{x} p-I_{x} q\right\|<\left\|\left(I-x A_{\epsilon_{n}}\right)^{-1} p-\left(I-x A_{\epsilon_{n}}\right)^{-1} q\right\|+\epsilon .
$$

Since $\left(I-x A_{\epsilon_{n}}\right)^{-1}$ is nonexpansive, it follows that $\left\|I_{x} p-I_{x} q\right\|<\|p-q\|$ $+\epsilon$. Thus $I_{x}$ is nonexpansive, and (i) of Theorem 2 is proved.

Lemma 9. For each $p$ in $S, G_{p}$ is continuous on $(0, \infty)$.

Proof. Suppose $p$ is in $S$. Since for each $x>0, G_{p}(x)$ is the sequential limit of $\left\{F_{p, i}(x)\right\}_{i=1}^{\infty}$, and since the set $\left\{F_{p, i} \mid i=1,2, \cdots\right\}$ is equicontinuous on $(0, \infty)$, it follows that $G_{p}$ is continuous on $(0, \infty)$.

Since for each $p$ in $S$ and each $x \geqq 0, I_{x} p=G_{p}(x)$, it follows from Lemma 9 that for $x>0$ and $p$ in $S, \lim _{y \rightarrow x} I_{y} p=I_{x} p$, and so (ii) of Theorem 2 is proved.

All of the above lemmas would still be true if, instead of assuming that $S$ contains a rest point, it is assumed that for each point $p$ in $S$, $\left\{\left\|\left(I-x A_{\delta}\right)^{-1} p\right\| \| \delta>0, x \geqq 0\right\}$ is bounded above. Requiring this boundedness is a weaker condition than requiring a rest point, since if $S$ contains a rest point, the above set is bounded above. However, to assume $S$ contains a rest point does not appear to be a very strong condition, since if there is at least one point $p$ in $S$ such that $g_{p}$ is not one-to-one, then $S$ contains a rest point. For if $g_{p}(u)=g_{p}(v)$ where $0 \leqq u<v$, then setting $q=g_{p}(u)$ it follows that

$$
\begin{aligned}
g_{q}(v-u) & =T(v-u)_{q}=T(v-u) g_{p}(u) \\
& =T(v-u) T(u) p=T(v) p=g_{p}(v)=q .
\end{aligned}
$$


Then, since $g_{q}(v-u)=q$ and $v-u>0, g_{q}$ is said to be periodic of period $v-u$, and the following result [5] of the author can be used to show that $(1 /(v-u)) \int_{0}^{v-u} g_{q}$ is a rest point.

Theorem 4. Assume $S$ is a Hilbert space and Conditions (1), (2), and (3) are satisfied. If for some point $p$ in $S, g_{p}$ is periodic of period $\epsilon>0$, then $(1 / \epsilon) \int_{0}^{\epsilon} g_{p}$ is a rest point.

For Lemma 10, Condition (5) will also be assumed. For Lemma 10, let $E$ denote a sequence $\left\{\epsilon_{i}\right\}_{i=1}^{\infty}$ of positive numbers converging to 0 such that for every $x \geqq 0$ and every $p$ in $S,\left\{\left(I-x A_{e_{i}}\right)^{-1} p\right\}_{i-1}^{\infty}$ converges, and let $I_{x} p$ be the sequential limit. Then, by Lemma 8 , for each $x \geqq 0, I_{x}$ is nonexpansive.

Lemma 10. Suppose $y>0$ and $p$ is in $S$. If $\epsilon>0$, there is $a \delta>0$ such that if $0=t_{0}<t_{1}<t_{2}<\cdots<t_{n+1}=y$ and $\left|t_{i+1}-t_{i}\right|<\delta$ for $i=0,1, \cdots$, $n$, then

$$
\left\|\prod_{i=0}^{n} I_{t_{i+1}-t_{i}} p-T(y) p\right\|<\epsilon .
$$

Proof. Let $\epsilon>0$. Using Theorem 1 , let $\delta$ be a positive number such that if $0=t_{0}<t_{1}<\cdots<t_{n+1}=y$ and $\left|t_{i+1}-t_{i}\right|<\delta$ for $i=0,1,2, \cdots, n$, then

$$
\underset{\delta_{0}, \delta_{1}, \cdots, \delta_{n} \rightarrow 0}{\lim \sup ^{-}}\left\|\prod_{i=0}^{n}\left[I-"\left(t_{i+1}-t_{i}\right) A_{\delta_{i}}\right]^{-1} p-T(y) p\right\|<\epsilon / 2 .
$$

Take $0=t_{0}<t_{1}<\cdots<t_{n+1}=y$ where $\left|t_{i+1}-t_{i}\right|<\delta$ for $i=0,1, \cdots, n$. There is a positive number $k$ such that if each of $\delta_{0}, \delta_{1}, \cdots, \delta_{n}$ is less than $k$, then

$$
\left\|\prod_{i=0}^{n}\left[I-\left(t_{i+1}-t_{i}\right) A_{\delta_{i}}\right]^{-1} p-T(y) p\right\|<\epsilon / 2 .
$$

Now $I_{t_{n+1}-t_{n}} p=\lim _{m \rightarrow \infty}\left[I-\left(t_{n+1}-t_{n}\right) A_{\epsilon_{m}}\right]^{-1} p$, and so $\delta_{n}$ can be chosen to be a number in the sequence $E$ which is less than $k$ and such that

$$
\left\|\left[I-\left(t_{n+1}-t_{n}\right) A_{\delta_{n}}\right]^{-1} p-I_{t_{n+1}-t_{n}} p\right\|<\epsilon / 2(n+1) .
$$

Since $I_{t_{n}-t_{n-1}}$ is nonexpansive, it follows that

(1) $\left\|I_{t_{n}-t_{n-1}}\left[I-\left(t_{n+1}-t_{n}\right) A_{\delta_{n}}\right]^{-1} p-I_{t_{n}-t_{n-1}} I_{t_{n+1}-t_{n}} p\right\|<\epsilon / 2(n+1)$.

Now

$$
\begin{aligned}
I_{t_{n}-t_{n-1}}[I & \left.-\left(t_{n+1}-t_{n}\right) A_{\delta_{n}}\right]^{-1} p \\
& =\lim _{m \rightarrow \infty}\left[I-\left(t_{n}-t_{n-1}\right) A_{e_{m}}\right]^{-1}\left[I-\left(t_{n+1}-t_{n}\right) A_{\delta_{n}}\right]^{-1} p
\end{aligned}
$$


and so $\delta_{n-1}$ can be chosen to be a number in sequence $E$ which is less than $k$ and such that

$$
\begin{aligned}
\|\left[I-\left(t_{n}-t_{n-1}\right) A_{\delta_{n-1}}\right]^{-1}\left[I-\left(t_{n+1}-t_{n}\right) A_{\delta_{n}}\right]^{-1} p \\
-I_{t_{n}-t_{n-1}}\left[I-\left(t_{n+1}-t_{n}\right) A_{\delta_{n}}\right]^{-1} p \|<\epsilon / 2(n+1) .
\end{aligned}
$$

From (1) and (2) it follows that

$$
\begin{gathered}
\left\|I_{t_{n}-t_{n-1}} I_{t_{n+1}-t_{n}} p-\left[I-\left(t_{n}-t_{n-1}\right) A_{\delta_{n-1}}\right]^{-1}\left[I-\left(t_{n+1}-t_{n}\right) A_{\delta_{n}}\right]^{-1} p\right\| \\
<2 \epsilon / 2(n+1) .
\end{gathered}
$$

Since $I_{t_{n-1}-t_{n-2}}$ is nonexpansive, it follows that

$$
\begin{aligned}
\| I_{t_{n-1}-t_{n-2}} I_{t_{n}-t_{n-1}} I_{t_{n+1}-t_{n}} p & \\
& \quad-I_{t_{n-1}-t_{n-2}}\left[I-\left(t_{n}-t_{n-1}\right) A_{\delta_{n-1}}\right]^{-1}\left[I-\left(t_{n+1}-t_{n}\right) A_{\delta_{n}}\right]^{-1} p \| \\
< & 2 \epsilon / 2(n+1) .
\end{aligned}
$$

Now

$$
\begin{aligned}
& I_{t_{n-1}-t_{n-2}} {\left[I-\left(t_{n}-t_{n-1}\right) A_{\delta_{n-1}}\right]^{-1}\left[I-\left(t_{n+1}-t_{n}\right) A_{\delta_{n}}\right]^{-1} p } \\
&=\lim _{m \rightarrow \infty}\left[I-\left(t_{n-1}-t_{n-2}\right) A_{\epsilon_{m}}\right]^{-1}\left[I-\left(t_{n}-t_{n-1}\right) A_{\delta_{n-1}}\right]^{-1} \\
& \cdot\left[I-\left(t_{n+1}-t_{n}\right) A_{\delta_{n}}\right]^{-1} p .
\end{aligned}
$$

Let $\delta_{n-2}$ be a number in sequence $E$ which is less than $k$ and such that

$$
\begin{aligned}
& \|\left[I-\left(t_{n-1}-t_{n-2}\right) A_{\delta_{n-2}}\right]^{-1}\left[I-\left(t_{n}-t_{n-1}\right) A_{\delta_{n-1}}\right]^{-1}\left[I-\left(t_{n+1}-t_{n}\right) A_{\delta_{n}}\right]^{-1} p \\
& -I_{t_{n-1}-t_{n-2}}\left[I-\left(t_{n}-t_{n-1}\right) A_{\delta_{n-1}}\right]^{-1}\left[I-\left(t_{n+1}-t_{n}\right) A_{\delta_{n}}\right]^{-1} p \| \\
& <\epsilon / 2(n+1) .
\end{aligned}
$$

From (3) and (4) it follows that

$$
\begin{aligned}
& \| I_{t_{n-1}-t_{n-2}} I_{t_{n}-t_{n-1}} I_{t_{n+1}-t_{n}} p-\left[I-\left(t_{n-1}-t_{n-2}\right) A_{\delta_{n-2}}\right]^{-1} \\
& \cdot\left[I-\left(t_{n}-t_{n-1}\right) A_{\delta_{n-1}}\right]^{-1}\left[I-\left(t_{n+1}-t_{n}\right) A_{\delta_{n}}\right]^{-1} p \| \\
& <3 \epsilon / 2(n+1) .
\end{aligned}
$$

Continuing this process of choosing $\delta_{n}, \delta_{n-1}, \cdots, \delta_{0}$, it can be seen that

(5) $\left\|\prod_{i=0}^{n} I_{t_{i+1}-t_{i}} p-\prod_{i=0}^{n}\left[I-\left(t_{i+1}-t_{i}\right) A_{\delta_{i}}\right]^{-1} p\right\|<(n+1) \epsilon / 2(n+1)$.

But each of $\delta_{0}, \delta_{1}, \cdots, \delta_{n}$ is less than $k$ and thus

$$
\left\|\prod_{i=0}^{n}\left[I-\left(t_{i+1}-t_{i}\right) A_{\delta_{i}}\right]^{-1} p-T(y) p\right\|<\epsilon / 2 .
$$


From (5) and (6) it follows that

$$
\left\|\prod_{i=0}^{n} I_{t_{i+1}-\iota_{1}} p-T(y) p\right\|<\epsilon
$$

and the lemma is proved.

It follows from Lemma 10 that $\lim _{n \rightarrow \infty}\left(I_{y / n}\right)^{n} p=T(y) p$, and thus (iii) of Theorem 2 is proved.

\section{REFERENCES}

1. E. Hille and R. S. Phillips, Functional analysis and semi-groups, rev. ed., Amer. Math. Soc. Colloq. Publ., Vol. 31, Amer. Math. Soc., Providence, R. I., 1957.

2. J. W. Neuberger, An exponential formula for one-parameter semi-groups of nonlinear transformations, J. Math. Soc. Japan 18 (1966), 154-157.

3. - A product integral formula for one-parameter semi-groups of nonlinear transformations, Abstract 66T-428, Notices Amer. Math. Soc. 13 (1966), 719.

4. Shinnosuke Ôharu, Note on the representation of semi-groups of nonlinear operators, Proc. Japan Acad. 42 (1966), 1149-1154.

5. Dorothy Rutledge, Semigroups of nonlinear transformations with periodic trajectories, Abstract 66T-347, Notices Amer. Math. Soc. 13 (1966), 622.

Agnes Scott College 